\title{
Exploration of a Multi-Parameter Technology for Pain Assessment in Postoperative Patients After Cardiac Surgery in the Intensive Care Unit: The Nociception Level Index (NOL) ${ }^{\mathrm{TM}}$
}

\author{
Céline Gélinas (D) $^{1,2}$ \\ Melissa Richard-Lalonde ${ }^{1,2}$ \\ Denny Laporta ${ }^{3,4}$ \\ Jean-François Morin ${ }^{5,6}$ \\ Madalina Boitor ${ }^{7}$ \\ Catherine E Ferland ${ }^{8,9}$ \\ Patricia Bourgault ${ }^{10}$ \\ Philippe Richebé (iD) ${ }^{11}$ \\ 'Ingram School of Nursing, McGill \\ University, Montreal, QC, Canada; ${ }^{2}$ Centre \\ for Nursing Research and Lady Davis \\ Institute, Jewish General Hospital - CIUSSS \\ West-Central Montreal, Montreal, QC, \\ Canada; ${ }^{3}$ Faculty of Medicine, Respiratory \\ Division, McGill University, Montreal, QC, \\ Canada; ${ }^{4}$ Department of Medicine, Division \\ of Adult Critical Care, Jewish General \\ Hospital - CIUSSS West-Central Montreal, \\ Montreal, QC, Canada; ${ }^{5}$ Faculty of \\ Medicine, Department of Surgery, Division \\ of Cardiac Surgery, McGill University, \\ Montreal, QC, Canada; ${ }^{6}$ Department of \\ Surgery, Cardiac Surgery, Jewish General \\ Hospital - CIUSSS West-Central Montreal, \\ Montreal, QC, Canada; ${ }^{7}$ Faculty of \\ Dentistry, McGill University, Montreal, QC \\ Canada; ${ }^{8}$ Faculty of Medicine, Department \\ of Anesthesia, McGill University, Montreal, \\ QC, Canada; ' Shriners Hospitals for \\ Children - Canada, Montreal, QC, Canada \\ ${ }^{10}$ Faculty of Medicine and Health Sciences, \\ School of Nursing, Université de \\ Sherbrooke, Sherbrooke, QC, Canada; \\ "Department of Anesthesiology and Pain \\ Medicine, Maisonneuve-Rosemont \\ Hospital - CIUSSS de l'Est-de-l'Île-de- \\ Montréal - Université de Montréal, \\ Montreal, QC, Canada
}

Correspondence: Céline Gélinas Ingram School of Nursing - McGill

University, 680, Sherbrooke West, Suite

I800, Montreal, QC, H3A 2M7, Canada

Tel + I 5 I4 3986157

Fax + I 5143988455

Email celine.gelinas@mcgill.ca
Introduction: The aim of this study was to explore the use of a multi-parameter technology, the Nociception Level (NOL) index (Medasense Biometrics Ltd, Ramat Gan, Israel), for pain assessment in postoperative awake patients after cardiac surgery during non-nociceptive and nociceptive procedures in the intensive care unit (ICU).

Materials and Methods: A prospective cohort repeated-measures design was used. Patients were included if they were in the ICU after undergoing cardiac surgery and if they could self-report their pain. A non-invasive probe was placed on the patient's finger for the continuous monitoring of the NOL index. Patients' self-reports of pain and anxiety $(0-10$ Numeric Rating Scale or NRS), and behavioral scores with the Critical-Care Pain Observation Tool (CPOT) were obtained before and during a non-nociceptive procedure (ie, non-invasive blood pressure [NIBP] using cuff inflation), and before, during and after a nociceptive procedure (ie, chest tube removal [CTR]) for a total of five time points. Nonparametric tests were used to compare scores at different time points, and receiver operating characteristic curve analysis was performed.

Results: Fifty-four patients were included in the analysis. The NOL index, pain and anxiety scores were significantly higher during CTR compared to rest and NIBP ( $p<0.001$ ). During CTR, the NOL was associated with self-reported pain intensity and unpleasantness but not with anxiety and CPOT scores. The NOL showed a modest performance in detecting pain (NRS $\geq 1$ and $\geq 5$ ) in this sample with sensitivity and specificity ranging from $61 \%$ to $85 \%$.

Conclusion: The NOL index was able to discriminate between a non-nociceptive and a nociceptive procedure and was associated with self-reported pain. Further validation testing of the NOL is necessary in a heterogeneous sample of ICU patients.

Keywords: nociception, pain, pain assessment, adult, cardiac surgery, intensive care unit

\section{Introduction}

Assessing pain in the intensive care unit (ICU) is a challenging endeavor for clinicians striving to provide the best care for their patients. The patient's selfreport should be obtained whenever possible as it represents the gold standard measure of pain. ${ }^{1}$ However, a significant proportion of ICU patients are unable to communicate due to their critical condition and related treatment. In such situations, behavioral scales such as the Critical-Care Pain Observation Tool (CPOT) ${ }^{2}$ are recommended alternative measures, but their use have limitations., ${ }^{3,4}$ Indeed, 
behaviors are impossible to monitor in paralyzed patients receiving neuromuscular blocking agents and they may be blurred with deep sedation. ${ }^{5}$ Therefore, it is necessary to explore other pain or nociception assessment methods. ${ }^{3-5}$

Physiological parameters such as vital signs (eg, blood pressure, heart rate and respiratory rate) and number of skin conductance fluctuations (NSCF) have been found to increase during nociceptive stimuli known to be painful. ${ }^{5-7}$ However, these parameters are not consistently related to self-reported pain by ICU patients. Previous studies using single physiological parameters have shown inter-individual variability to be a major limiting factor in the fields of anaesthesiology and pain. ${ }^{8}$ The multi-parameter Nociception Level (NOL) index incorporates simultaneously heart rate, photoplethysmography and skin conductance parameters to estimate nociception levels. ${ }^{9}$ The combination of these parameters significantly differentiated between low, moderate and high heat pain intensities in 45 healthy subjects. ${ }^{10}$ The ability of the NOL index to classify nociceptive versus non-nociceptive stimuli was high with an area under the curve (AUC) $>0.90$ in anaesthetized patients undergoing surgery. ${ }^{11-13}$ Also, NOLguided anesthesia resulted in lower pain scores in the postoperative phase, ${ }^{14}$ and a $30 \%$ reduction in intraoperative opioid use. ${ }^{15}$

However, the use of this multi-parameter technology is new to the ICU context. To our knowledge, only our previous pilot study on the NOL's validation with 15 intubated ICU patients has been recently published. ${ }^{16}$ This pilot testing has shown that the NOL could discriminate between a nociceptive (endotracheal suctioning) and a non-nociceptive (non-invasive blood pressure [NIBP] using cuff inflation) procedure (Wilcoxon signed-rank test, $\mathrm{p}<0.001$ ), and the NOL index was positively associated with self-reported pain intensity and CPOT scores (Mann-Whitney $U$ tests, $\mathrm{p}<0.05$ ). The present study aimed to pursue validation testing using a different standard of care procedure, namely the chest tube removal (CTR), which is one of the most painful procedures reported after cardiac surgery by ICU patients. ${ }^{6,17}$

\section{Objectives}

This study aimed to explore the use of the NOL index, a multi-parameter technology, for pain assessment in postoperative cardiac surgery ICU adults able to self-report. Primary validation objectives were to examine the use of the NOL index to:
1. Discriminate between nociceptive (CTR) and nonnociceptive (NIBP) procedures part of standard ICU care.

2. Detect self-reported pain intensity during CTR.

3. Describe its association with self-reports of pain intensity, pain unpleasantness and anxiety, and CPOT scores during CTR.

As a secondary objective, we described the feasibility of the NOL's use for research purposes in the ICU context.

\section{Materials and Methods \\ Design}

A prospective cohort design including repeated-measures was used for this study.

\section{Setting and Sample}

This study was conducted in a 24-bed medical-surgical ICU in Montreal, Canada. Patients were considered eligible if they 1) underwent elective cardiac surgery; 2) were adults (18 years or older); 3) spoke French or English or had a family member as an interpreter; and 4) were able to self-report. Patients were excluded if they suffered from any form of paralysis, psychiatric or neurological disease, and if the physiological signal acquired by the NOL device was not suitable for NOL index calculation. Having an intact motor function was essential as CPOT scores are dependent on behavioral reactions.

The study was powered to detect a moderate positive correlation $(>0.40)^{6}$ between the NOL index and selfreported pain intensity. A minimum of 47 patients was needed with an alpha error of 0.05 and a power of $80 \%$. Considering an attrition rate of $10 \%$ as found in previous studies of the principal investigator (CG), a sample of 52 participants was required. The sample size was reached using consecutive sampling. All patients admitted for elective cardiac surgery from February to August 2017 were screened for eligibility and were approached by the nursing staff to participate in the study when eligible.

\section{Procedures}

The Medical/Biomedical Research Ethics Committee of the Jewish General Hospital - CIUSSS West-Central Montreal approved this research project (CODIM-MBM -16-207). This study was conducted in accordance with the Declaration of Helsinki. The research staff met with eligible patients the day before surgery to explain the study 
procedures, obtain written informed consent and collect socio-demographic data (eg, age, sex). Consented patients were taught how to use the $0-10$ numeric rating scales (NRS) for pain intensity, pain unpleasantness and anxiety, and how the NOL non-invasive probe would be placed on their finger.

When the patients' condition allowed the planning of CTR, the research staff placed the non-invasive probe with the disposable adhesive electrodes on one of the patient's fingers (usually the index), set up the NOL device at the bedside for calibration, and turned the screen away from the patient to reduce any bias during self-reporting. A video camera was also installed at the foot of the bed to capture the patient's behaviors for interrater reliability of CPOT scores. A total of five assessment time points of each measure (NOL index, CPOT scores, and patients' self-reports of pain intensity, pain unpleasantness and anxiety) were completed by trained research staff while patients were in the ICU. Assessments were done at rest before and during a non-nociceptive procedure (ie, NIBP using cuff inflation), and at rest before, during, and 5 minutes after a nociceptive procedure (ie, CTR). For assessments during procedures, patients were asked to rate their symptoms experienced during the procedures right after they were completed. NIBP is easily accessible using the standard ICU monitoring equipment and has been shown to be painless in ICU adult patients. ${ }^{6,18}$ Assessments related to NIBP were performed first when possible. This procedure was done by the responsible ICU nurse or by the research staff under the supervision of the nurse. CTR was performed by the resident, fellow or cardiac surgeon.

At each time point, CPOT scoring was done by the research staff prior to obtaining the patient's self-report to avoid rater bias. CPOT assessments were done for a 60 second duration except for CTR, which lasted longer (up to 180 seconds) and included the dressing, stitches, and tubes removal. The NOL index was continuously recorded (ie, values generated every 5 seconds) during the whole data collection, and data were then extracted for data analysis in a standardized manner as done in previous studies in anesthesiology. ${ }^{12,13}$ More specifically, baseline NOL values were averaged over 1 minute when no stimulation was done (ie, before NIBP and CTR). During NIBP and within a 1-minute window after the start of cuff inflation, NOL values were averaged within 15 seconds before and after the peak value obtained during this nonnociceptive procedure. NOL values were averaged in a similar way during CTR, ie, within 15 seconds before and after the peak value obtained during a 180 -seconds window after the start of this nociceptive procedure. At the end of data collection, the research staff collected type of cardiac surgery, cardiac medication, opioid analgesia and sedation administration within 4 hours pre-procedure and within 1 hour prior to CTR from the patients' medical charts. All medications were administered by the nurses according to the patients' needs and as prescribed by the physicians. Videos were viewed by a trained rater not involved in CPOT scoring at the bedside at least 1 month after data collection to check for interrater reliability of CPOT scores with the bedside rater.

\section{Instruments}

Nociception Level (NOL) Index

The NOL index PMD200 ${ }^{\mathrm{TM}}$ (Medasense Biometrics Ltd, Ramat Gan, Israel) simultaneously incorporates the heart rate, heart rate variability $(0.15-0.4 \mathrm{~Hz}$ band power), photoplethysmographic waveform amplitude, skin conductance level, NSCF, and their time derivatives, using a nonlinear Random Forest regression technique to estimate the nociception or related pain level. ${ }^{9}$ The NOL index is a single number from 0 to 100 obtained through a noninvasive finger probe. Values above 25 are believed to be indicative of nociception and related pain.

\section{Numeric Rating Scale (NRS)}

The patient's self-reports of pain intensity, pain unpleasantness and anxiety were assessed using 0 to 10 NRS. ${ }^{19}$ For pain intensity, the Faces Pain Thermometer (FPT), which is a thermometer including six faces with NRS scoring from 0 "no pain" to 10 "worst possible pain," was used. ${ }^{20}$ For pain unpleasantness, the NRS from 0 "not at all unpleasant" to 10 "most unpleasant feeling possible" was utilized. The 0-10 NRS (0 for "no anxiety" and 10 for "worst possible anxiety") was also selected to remain consistent with the format of self-reports for pain intensity and unpleasantness, and thereby, to facilitate patients' scoring of symptoms.

\section{Critical-Care Pain Observation Tool (CPOT)}

The CPOT includes four behavioral items: 1) facial expression, 2) body movements, 3) muscle tension, and 4) compliance with the ventilator (for intubated patients) or vocalization (for those non-intubated). ${ }^{2}$ Each item is rated on a scale from 0 to 2 for a total CPOT score ranging from 0 to 8 . The $\mathrm{CPOT}$ has shown robust 
reliability and validity findings in cardiac surgery and other ICU patients. ${ }^{2,6,21}$ It was found to be significantly correlated with self-reported pain intensity $(r>0.40)$ and pain unpleasantness $(r>0.30)$, and to increase during nociceptive procedures when compared with rest or nonnociceptive procedures (CPOT scores $\geq 2$ ).

\section{Socio-Demographic and Clinical Variables}

Socio-demographic (ie, age, sex) and clinical data including type of cardiac surgery, number of chest tubes in place, sedation level based on the Richmond Agitation Sedation Scale scores (RASS), ${ }^{22}$ cardiac medication, administered opioids and sedatives were also collected from the medical charts.

\section{Data Analysis}

Descriptive statistics (frequencies and percentages for categorical data; medians, ranges and/or interquartile ranges [IQR] for continuous data) were computed for all study variables at each time point. Intraclass correlation coefficient (ICC) was computed to determine the interrater reliability of CPOT scores between the two raters at each time point.

Given that variables were not normally distributed (Shapiro-Wilk Test, $\mathrm{p}<0.05$ and kurtosis and skewness indices $> \pm 2)^{23}$ at most time points (ie, before and during NIBP, post CTR), nonparametric tests were used. NOL index values were compared across time points and procedures (ie, CTR, NIBP) (Friedman and Wilcoxon signed-rank tests) to examine its ability to discriminate between conditions. NOL index values were also compared across patient groups (eg, with or without pain, different pain levels) (Kruskal-Wallis and Mann-Whitney $U$ tests) to explore its ability to detect pain during CTR. The receiver operating curve (ROC) analysis was also performed to estimate the Area Under the Curve (AUC), sensitivity and specificity of the NOL index to detect pain during CTR. Associations between all variables, including the NOL index, self-reports of pain intensity, pain unpleasantness and anxiety as well as CPOT scores were examined during CTR using the Spearman's rho correlation or the point-biserial correlation. CPOT scores of the bedside rater were used for data analysis. The alpha error was initially set at 0.05 and was adjusted to 0.017 for post-hoc MannWhitney $U$ and Wilcoxon signed-rank tests to account for multiple tests based on Bonferroni correction. Calibration time, challenges encountered with the NOL device and finger probe and mitigation strategies described the feasibility of the NOL's use for research purposes in the ICU context.

\section{Results}

\section{Sample of Participants}

A total of 118 patients were screened and approached for inclusion in this study, and 96 provided informed consent. From these, 11 had their surgery cancelled, and 1 returned to his home country. In addition, one patient withdrew, three did not have their chest tube removed in the ICU, four could not self-report their pain, and six had their chest tube removed without the research team being notified. Therefore, data collection was attempted for 70 patients; however, photoplethysmography (PPG) signal quality issues of the NOL index occurred for 16 patients. This was mainly due to a technical fault of the finger probe, which has been caused by mechanical damage. The finger probe was therefore replaced and the PPG performed in a satisfactory manner afterwards. These 16 patients were mostly male (81\%) with a mean age of 65 years old $(\mathrm{SD}=9.90)$ and underwent coronary artery bypass graft (CABG) surgery $(75 \%)$. They were not significantly different in sociodemographic and medical data compared to the patients included in the data analysis $(p>0.45)$. A total of 54 patients were included in the final data analysis. Most participants were male (72\%), with a mean age of 66 years old, underwent CABG (61\%), and had an average of three chest tubes (including mediastinal and pleural drains) removed on postoperative day 1 or 2 after the surgery. One-third of the patients (33\%) did not receive any opioid analgesia and the majority did not receive any sedatives (94\%) within 4 hours prior to CTR (Table 1). A total of 10 patients were administered cardiac medication. More specifically, four patients received PO Metoprolol, two of them received PO Bisoprolol, and one received PO Amlodipine. Only three patients were on low doses of IV infusions, ie, two on dobutamine and one on norepinephrine bitartrate (Levophed) and milrinone.

\section{Description of Pain and Anxiety Scores at Each Time Point}

Before NIBP, pain intensity and pain unpleasantness were of mild intensity, and anxiety and CPOT were at the lowest scores (Table 2). During NIBP, all scores were at their lowest value. Before CTR, pain intensity and anxiety were of mild intensity, and other scores were at zero. All values increased during CTR to reach moderate values (NRS $>4$ ) and reached CPOT scores $>2$. After CTR, all scores decreased and reached mild values (NRS $\leq 3$ ) or 0 scores. These changes were statistically significant for all pain and anxiety variables. 
Table I Socio-Demographic and Medical/Surgical Data for Participating Patients

\begin{tabular}{|c|c|}
\hline Characteristics & $\begin{array}{l}\text { Descriptive Statistics } \\
(n=54)\end{array}$ \\
\hline Age (mean, standard deviation) & $65.76(11.77)$ \\
\hline \multicolumn{2}{|l|}{$\operatorname{Sex}(n, \%)$} \\
\hline Male & $39(72.2)$ \\
\hline Female & $15(27.8)$ \\
\hline \multicolumn{2}{|l|}{ Cardiac surgery $(\mathrm{n}, \%)$} \\
\hline CABG & $33(6 I . I)$ \\
\hline CABG and valve replacement & $5(9.3)$ \\
\hline Valve replacement & $12(22.2)$ \\
\hline Valve repair & $2(3.7)$ \\
\hline Valve replacement and repair & I (I.9) \\
\hline Ascending aorta replacement & I (I.9) \\
\hline Ejection fraction \% (median, range) & $60(25-75)$ \\
\hline $\begin{array}{l}\text { Number of days since surgery (median, } \\
\text { range) }\end{array}$ & $I(I-4)$ \\
\hline $\begin{array}{l}\text { Richmond-Agitation Sedation Scale } \\
\text { (median, range) }\end{array}$ & $0(-I$ to $I)$ \\
\hline $\begin{array}{l}\text { Number of chest tubes removed } \\
\text { (median, range) }\end{array}$ & $3(1-4)$ \\
\hline \multicolumn{2}{|l|}{ Opioid analgesia within I hour of CTR (n, \%) } \\
\hline Yes & $10(18.5)$ \\
\hline No & $44(81.5)$ \\
\hline $\begin{array}{l}\text { Equianalgesia within I hour of CTR (mg } \\
\text { of Morphine per os) }(n=10) \text { (median, } \\
\text { range) }\end{array}$ & $25.00(12.5-25.00)$ \\
\hline \multicolumn{2}{|l|}{$\begin{array}{l}\text { Opioid analgesia within } 4 \text { hours of CTR } \\
(n, \%)\end{array}$} \\
\hline Yes & $36(66.7)$ \\
\hline No & $18(33.3)$ \\
\hline $\begin{array}{l}\text { Equianalgesia within } 4 \text { hours of CTR (mg } \\
\text { of Morphine per os) }(n=36) \text { (median, } \\
\text { range) }\end{array}$ & $25.00(7.50-75.00)$ \\
\hline \multicolumn{2}{|l|}{ Sedation within 4 hours of CTR (n, \%) } \\
\hline Yes & $\begin{array}{l}3 \text { (Propofol } n=2 ; \\
\text { Dexmedetomidine } n=1 \text { ) } \\
(5.6)\end{array}$ \\
\hline No & $51(94.4)$ \\
\hline
\end{tabular}

Abbreviations: CABG, coronary artery bypass graft; CTR, chest tube removal.

\section{Interrater Reliability of CPOT Scores}

Interrater reliability of CPOT scores between the two raters (one at the bedside and one who viewed videos) was supported with high ICC $(>0.80)^{24}$ for most time points. ICC before and during NIBP were 0.88 (95\% CI: $0.81-0.93)$ and 0.91 (95\%
CI: 0.85-0.94), respectively. A lower acceptable ICC was obtained before CTR with a value of 0.73 (95\% CI: 0.58 0.83). ICC during CTR and 5 minutes after were high with values of 0.83 (95\% CI: $0.73-0.89)$ and 0.89 (95\% CI: 0.82 $0.93)$, respectively.

\section{The NOL Index Discriminating Nociceptive from Non-Nociceptive Procedures}

The NOL index (Table 2) was significantly higher during CTR compared to pre-procedure (Wilcoxon signed-rank test $=4.97, \mathrm{p}<0.001$ ) and post-procedure (Wilcoxon signed-rank test $=5.74, \mathrm{p}<0.001)$. The NOL index was also significantly higher during CTR than during NIBP (Wilcoxon signed-rank test $=5.42, \mathrm{p}<0.001)$. The NOL index did not significantly increase during NIBP (Wilcoxon signed-rank test $=-1.94$, $\mathrm{p}<0.05$ ). One out of three patients did not receive any opioid within 4 hours prior to CTR. No difference in mean ranks was found for the NOL index between patients who received opioids within 4 hours prior to CTR and those who did not (Mann-Whitney $U$ test $=279.00, \mathrm{p}=0.842$ ).

\section{The NOL Index for Pain Detection}

Among the patients with a calculable NOL index during CTR $(\mathrm{n}=51), 4$ patients reported no pain $(\mathrm{NRS}=0), 14$ reported mild pain (NRS $=1-4$ ) and 33 reported moderate-to -severe pain (NRS $\geq 5$ ). Descriptive statistics of the NOL and CPOT for each pain level are reported in Table 3. The NOL index was different across the three pain levels (KruskalWallis test $=6.00, \mathrm{p}=0.05)$. We found that the NOL index tended to be higher for patients with moderate-to-severe pain than for those with mild pain (Mann-Whitney $U$ test = $151.00, \mathrm{p}=0.063$ ) and those with no pain (Mann-Whitney Utest $=27.00, \mathrm{p}=0.056)$. The ROC analysis using selfreported pain intensity $\geq 1$ and $\geq 5$ as reference criteria during CTR indicated an AUC $\geq 0.70$ with sensitivity $\geq 75 \%$ and specificity $>60 \%$ (Figure 1). Best NOL cut-off values were $\geq 22$ and $\geq 26$ with self-reported pain intensity thresholds $\geq 1$ and $\geq 5$, respectively. Negative predictive value (NPV) was higher $(74 \%)$ than positive predictive value ( $\mathrm{PPV}<60 \%)$ at both self-reported pain intensity thresholds.

\section{Associations Between Variables During the Nociceptive Procedure}

During CTR, CPOT scores were moderately associated with self-reported pain intensity, pain unpleasantness, and anxiety scores. Self-reported pain intensity, pain 
Table 2 Medians and Interquartile Ranges (IQR) for Patients' Self-Reports of Pain Intensity, Pain Unpleasantness, Anxiety NRS scores; CPOT scores and NOL index values Across Procedures

\begin{tabular}{|l|l|l|l|l|l|l|l|}
\hline Outcome & Before NIBP & During NIBP & $\begin{array}{l}\text { Wilcoxon } \\
\text { Signed-Rank } \\
\text { Test }\end{array}$ & Before CTR & During CTR & $\begin{array}{l}\text { 5-Minutes } \\
\text { After CTR }\end{array}$ & $\begin{array}{l}\text { Friedman } \\
\text { Test }\end{array}$ \\
\hline Pain intensity & $3(0.5-5)$ & $0(0-2)$ & $-4.07^{* * *}$ & $2(0-5)$ & $6(3-9)$ & $3(0-5)$ & $46.39^{* * *}$ \\
Pain unpleasantness & $2(0-6)$ & $0(0-2)$ & $-3.28^{* * *}$ & $0(0-6)$ & $4.5(0.5-8)$ & $1(0-5.5)$ & $14.31^{* * *}$ \\
Anxiety & $0(0-4.25)$ & $0(0-1)$ & $-2.89 * *$ & $1(0-6)$ & $5.5(0-10)$ & $0(0-5)$ & $22.20^{* * *}$ \\
CPOT & $0(0-0)$ & $0(0-0)$ & 0.00 & $0(0-1)$ & $3(1-4)$ & $0(0-1)$ & $74.38^{* * *}$ \\
NOL index & $5.55(3.00-16.40)$ & $10.74(5.22-17.20)$ & $-1.94^{*}$ & $19.11(7.64-26.16)$ & $28.90(23.23-35.74)$ & $8.62(2.76-15.82)$ & $42.87^{* * *}$ \\
\hline
\end{tabular}

Notes: ${ }^{*} p<0.05$. ** $p<0.01$. **** $<0.001$.

Abbreviations: NRS, Numeric Rating Scale; NIBP, non-invasive blood pressure; CTR, chest tube removal; CPOT, Critical-Care Pain Observation Tool; NOL, Nociception Level.

Table 3 Medians and Interquartile Ranges (IQR) of the NOL index and CPOT at Different Pain Intensity Levels During CTR

\begin{tabular}{|l|l|l|l|l|l|}
\hline \multirow{2}{*}{ NRS } & \multirow{3}{*}{$\mathbf{N}$} & \multicolumn{2}{|l|}{ NOL index } & \multicolumn{2}{l|}{ CPOT } \\
\cline { 3 - 6 } & Median & IQR & Median & IQR \\
\hline 0 & 4 & 20.50 & $17.58-32.23$ & I & $0.25-1.75$ \\
I-4 & 14 & 25.45 & $20.58-32.28$ & I & I-3 \\
$5-10$ & 33 & 31.99 & $25.43-36.29$ & 3 & $2-5$ \\
\hline
\end{tabular}

Abbreviations: NOL, Nociception Level; CPOT, Critical-Care Pain Observation Tool; CTR, chest tube removal.

unpleasantness and anxiety were moderately to highly associated with each other (Table 4). Point-biserial correlations of threshold values of NOL, pain and anxiety variables are presented in Table 5. The lowest NOL threshold $\geq 22$ was associated with pain intensity and pain unpleasantness $\geq 1$, but not with anxiety. A tendency towards a significant mild correlation was noted between the lowest NOL threshold and CPOT $\geq 2(\mathrm{p}=0.071)$. Similar results were obtained using the highest NOL threshold $\geq 26$ and moderate-to-severe symptom level $\geq 5$. This NOL threshold was associated with moderate-tosevere pain intensity and pain unpleasantness but not with anxiety and CPOT thresholds.

\section{Feasibility of the NOL's Use for Research Purposes in the ICU Context}

There were 70 instances when the device was calibrated throughout the data collection attempts. Although Medasense Ltd recommended a calibration time of 5 minutes (300 seconds) to obtain a stable signal, it took an average of 95.68 seconds (1.59 minutes) to obtain an initial NOL index signal $(\mathrm{SD}=213.94$; $\min =30$; $\max =$ 1585). The mean duration (in seconds) of an entire participant's NOL index recording was 2795.63 (46.6 minutes $) \pm 2351.55($ median $=2400 ; \min =510 ; \max =$
17,670). There was only one instance that the total time waiting to get a NOL index signal was 2760 seconds (46.0 minutes), but the device did not calibrate. It was a common occurrence that the probe had to be moved from one finger to another to get a NOL index signal. On eight separate occasions, the NOL index signal was lost at some point during the data collection process (signal loss duration ranged from 65 to 390 seconds). Artefacts in the data arose from multiple factors such as movements of the participants (including coughing, drinking water, talking, being talked to or repositioned). We used warm blankets around the arm and hand of participants with cold hands to obtain a NOL index signal. Other factors that interfered with proper NOL index signal were the size and shape of the participants' fingers (eg, too large to fit the probe or too contracted to have proper contact with electrodes).

\section{Discussion}

To our knowledge, this is one of the first studies evaluating the use of the NOL index in a population of postoperative ICU patients able to self-report. This study was primarily designed to detect a moderate positive correlation $(>0.40)$ between the NOL index and self-reported pain during a nociceptive procedure. We found positive mild correlations between the two NOL threshold values and pain intensity levels $\geq 1$ and $\geq 5$. Interestingly, there was no significant correlation between the NOL and anxiety, suggesting that the NOL index was not influenced by the anxiety level in this ICU sample during CTR. The NOL was also not correlated with the CPOT, which is a recommended alternative behavioral measure for pain in ICU patients unable to self-report.

The NOL was able to discriminate between the nociceptive and the non-nociceptive procedures with higher 
A

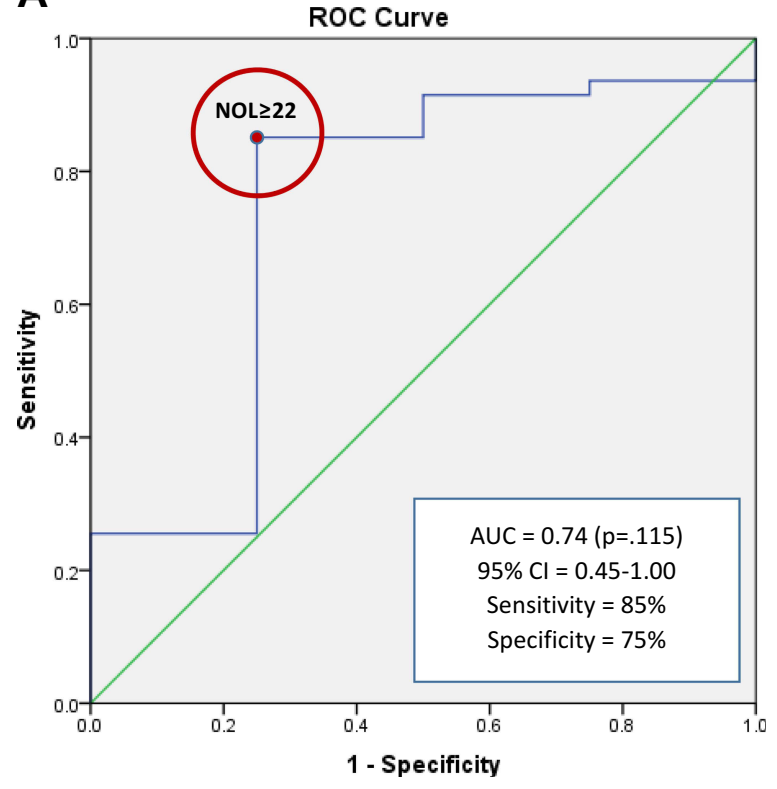

$$
\text { B }
$$

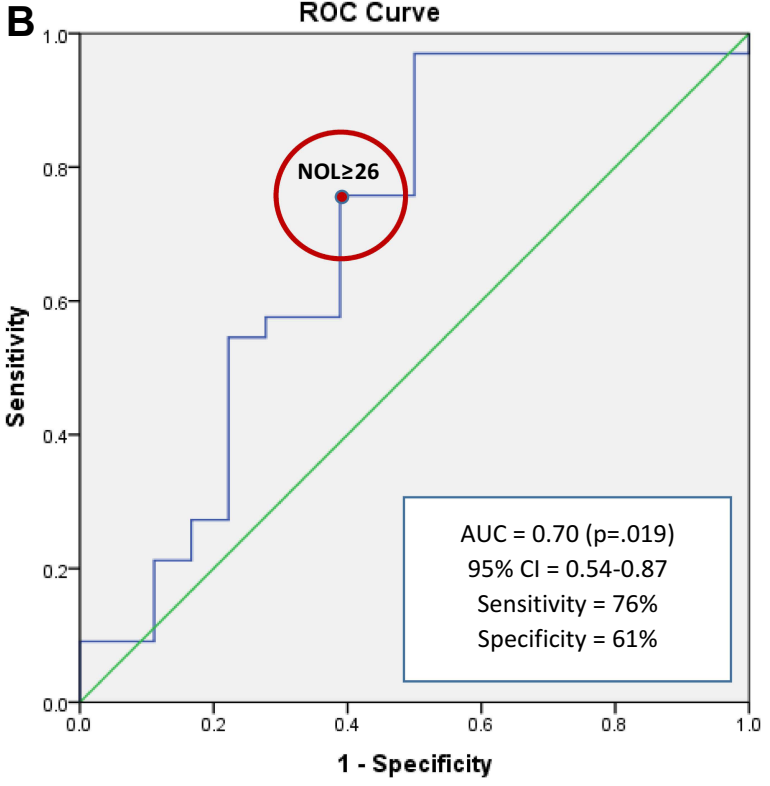

Figure I ROC analyses of the NOL index during CTR using 0-10 NRS thresholds of $I$ (a) and 5 (b) for pain intensity as reference criteria. (A) Self-reported pain intensity $\geq$ I. (B) Self-reported pain intensity $\geq 5$.

Abbreviations: AUC, area under the curve; $\mathrm{Cl}$, confidence interval; NRS, Numeric Rating Scale; CTR, chest tube removal; ROC, receiver operating curve.

values during CTR. This finding is consistent with previous studies in anaesthetized patients ${ }^{9,11-13,25-27}$ as well as in our pilot study ${ }^{16}$ comparing NOL values between NIBP and endotracheal suctioning in mechanically ventilated ICU patients. Interestingly, the NOL could also discriminate between three pain levels (no pain, mild pain, moderate-to-severe pain). The NOL index increased when patients reported higher pain levels. ROC analyses
Table 4 Spearman Rho Correlations of CPOT, Pain and Anxiety NRS Scores During CTR

\begin{tabular}{|l|l|l|l|}
\hline & $\begin{array}{l}\text { Pain } \\
\text { Intensity }\end{array}$ & $\begin{array}{l}\text { Pain } \\
\text { Unpleasantness }\end{array}$ & Anxiety \\
\hline CPOT & $0.52^{* * *}$ & $0.47^{* * *}$ & $0.46 * * *$ \\
Pain intensity & & $0.66^{* * *}$ & $0.72 * * *$ \\
Pain & & & $0.59 * * *$ \\
unpleasantness & & & \\
\hline
\end{tabular}

Note: $* * * p<0.001$.

Abbreviations: CPOT, Critical-Care Pain Observation Tool; NRS, Numeric Rating Scale; CTR, chest tube removal.

supported modest performance of the NOL in its ability to discriminate pain using two NRS thresholds ( $\geq 1$ and $\geq 5$ ) with acceptable to good sensitivity and specificity. The NOL threshold values were aligned with the manufacturer's recommendation and higher in this awake postoperative ICU sample compared to NOL threshold values established in anaesthetized patients using different reference standards. ${ }^{9,12,26}$ The NOL also showed a better NPV than PPV. This is a relevant finding considering that opioids should not be administered when their use is not required (ie, in the absence of significant pain).

As expected and found in previous studies, ${ }^{6,21}$ the CPOT was moderately associated with self-reported pain intensity supporting its criterion validation with the gold standard measure of pain. The correlation between CPOT and pain unpleasantness is reported for the second time with a slightly higher value compared to the previous finding $(r=0.31){ }^{6}$ A moderate association between CPOT and anxiety is reported for the first time showing that more intense painrelated behaviors are correlated with higher anxiety levels. Consistent with existing evidence in postoperative patients, anxiety and pain intensity were highly correlated. ${ }^{28}$

Contrary to our previous pilot findings, ${ }^{16}$ the present study could not demonstrate a significant association between CPOT and NOL index during the nociceptive procedure. This might be explained by the fact that CPOT and the NOL measure different components related to pain. The CPOT measures expressive behaviors related to pain, and the NOL measures physiological parameters that are involved in the nociception process, which may lead to pain. Therefore, the CPOT and the NOL likely provide complementary information in relation to the assessment of pain and nociception.

Regarding the feasibility of using the NOL index for research purposes in ICU patients able to self-report, this study reports short calibration time but also describes few challenges affecting the quality of the NOL signal and the 
Table 5 Point-Biserial Correlations Between NOL index, CPOT, Pain and Anxiety NRS Thresholds During CTR

\begin{tabular}{|c|c|c|c|c|}
\hline & СРОТ 2-8 & Pain Intensity $1-10$ & Pain Unpleasantness I-I0 & Anxiety $1-10$ \\
\hline \multirow[t]{2}{*}{ NOL $\geq 22$} & 0.26 & $0.4 I^{* *}$ & $0.31 *$ & 0.14 \\
\hline & СРОТ 3-8 & Pain Intensity 5-10 & Pain Unpleasantness 5-10 & Anxiety 5-10 \\
\hline NOL $\geq 26$ & 0.21 & $0.36 *$ & $0.29 *$ & 0.06 \\
\hline
\end{tabular}

Notes: $*_{p}<0.05$. ** $p<0.01$.

Abbreviations: CPOT, Critical-Care Pain Observation Tool; NRS, Numeric Rating Scale; CTR, chest tube removal.

one size of the finger probe not suited for all patients. Quality of the NOL signal was mainly affected by the patient's movements, and poor peripheral vascularization at the fingertip. Mitigation strategies included reminding patients to minimize movements during data collection and using warm blankets around their arm and hand. Similar challenges and mitigation strategies were reported in our previous pilot study, ${ }^{16}$ but rarely reported in other NOL studies in anaesthetized and paralyzed patients under controlled pulmonary ventilation. ${ }^{11-13,26}$ The updated PMD200 ${ }^{\mathrm{TM}}$ NOL technology and a new adjustable finger probe address these challenges and provide an adapted solution for ICU usage and should be utilized in future studies.

This study had some limitations. First, the homogeneous small sample limits the generalizability of findings to the broader ICU population. We made the decision to recruit postoperative ICU patients able to self-report to validate the NOL index with the gold standard measure of pain. Second, the $0-10$ NRS is not a common measure of anxiety, ${ }^{29}$ but we selected it for simplicity and consistency with self-report pain scales. Third, it was impossible to blind the CPOT raters to the type of procedure the participants underwent. Therefore, it is possible that raters perceived more intense behaviors during CTR known to be painful. Nonetheless, the examination of interrater reliability minimized this potential bias and it is unlikely that both raters were simultaneously biased. Finally, the preparation before CTR and interaction between the healthcare professional who removed the chest tubes and the patient could have influenced the pain experienced during the nociceptive procedure and could be explored in future studies.

\section{Conclusions}

1In summary, this study demonstrated promising findings of the NOL index for pain detection in the ICU. Indeed, the NOL index could discriminate between a non-nociceptive and a nociceptive procedure as well as between pain levels. Moreover, the NOL index was significantly associated with self-reported pain intensity and unpleasantness. Further validation of the NOL index with a heterogeneous sample of ICU patients including individuals unable to communicate who are deeply sedated or receiving neuromuscular blocking agents is needed to confirm the validity of its use in this population. Identifying a valid physiological measure of pain would fill a major gap in the recognition and detection of pain in the vulnerable population of ICU patients.

\section{Acknowledgments}

This study was funded by the Louise \& Alan Edwards Foundation and the Quebec Nursing Intervention Research Network. The PMD200 ${ }^{\mathrm{TM}}$ device was loaned by the company Medasense Ltd to the research team for the duration of this study. The funding agencies were not involved in the design, data analysis and write-up of this manuscript.

\section{Disclosure}

Dr Céline Gélinas reports grants from the Louise and Alan Edwards Foundation, Quebec Nursing Intervention Research Network, during the conduct of the study; free loan of PMD-200 NOL index device from Medasense Ltd, during the conduct of the study. Prof. Dr Philippe Richebé reports grants, personal fees from Medasense Ltd, outside of the submitted work. The authors report no other conflicts of interest in this work.

\section{References}

1. International Association for the Study of Pain (IASP). IASP terminology. Available from: https://www.iasp-pain.org/resources/ter minology/\#pain. Accessed November 24, 2021.

2. Gélinas C, Fillion L, Puntillo KA, Viens C, Fortier M. Validation of the critical-care pain observation tool in adult patients. Am J Crit Care. 2006;15(4):420-427. doi:10.4037/ajcc2006.15.4.420

3. Devlin JW, Skrobik Y, Gélinas C, et al. Clinical practice guidelines for the prevention and management of pain, agitation/ sedation, delirium, immobility, and sleep disruption in adult patients in the ICU. Crit Care Med. 2018;46(9):e825-e873. doi:10.1097/CCM.0000000000003299

4. Herr K, Coyne PJ, Ely E, Gélinas C, Manworren RCB. Pain assessment in the patient unable to self-report: clinical practice recommendations in support of the ASPMN 2019 position statement. Pain Manag Nurs. 2019;20(5):404-417. doi:10.1016/j.pmn.2019.07.005 
5. Gélinas C. Pain assessment in the critically ill adult: recent evidence and new trends. Intensive Crit Care Nurs. 2016;34:1-11. doi:10.1016/j.iccn.2016.03.001

6. Boitor M, Fiola JL, Gélinas C. Validation of the critical-care pain observation tool and vital signs in relation to the sensory and affective components of pain during mediastinal tube removal in postoperative cardiac surgery intensive care unit adults. J Cardiovasc Nurs. 2016;31(5):425-432. doi:10.1097/JCN.0000000000000250

7. Gunther AC, Bottai M, Schandl AR, Storm H, Rossi P, Sackey PV. Palmar skin conductance variability and the relation to stimulation, pain and the motor activity assessment scale in intensive care unit patients. Crit Care. 2013;17(2):R51. doi:10.1186/cc12571

8. Gruenewald M, Ilies C. Monitoring the nociception-anti-nociception balance. Best Pract Res Clin Anaesthesiol. 2013;27(2):235-247. doi:10.1016/j.bpa.2013.06.007

9. Ben-Israel N, Kliger M, Zuckerman G, Katz Y, Edry R. Monitoring the nociception level: a multi-parameter approach. J Clin Monit Comput. 2013;27(6):659-668. doi:10.1007/s10877-013-9487-9

10. Treister R, Kliger M, Zuckerman G, Aryeh IG, Eisenberg E. Differentiating between heat pain intensities: the combined effect of multiple autonomic parameters. Pain. 2012;153(9):1807-1814. doi:10.1016/j.pain.2012.04.008

11. Edry R, Recea V, Dikust Y, Sessler DI. Preliminary intraoperative validation of the nociception level index: a noninvasive nociception monitor. Anesthesiology. 2016;125(1):193-203. doi:10.1097/ ALN.0000000000001130

12. Renaud-Roy E, Stöckle PA, Maximos S, et al. Correlation between incremental remifentanil doses and the nociception level (NOL) index response after intraoperative noxious stimuli. Can J Anaesth. 2019;66(9):1049-1061. doi:10.1007/s12630-019-01372-1

13. Stöckle PA, Julien M, Issa R, et al. Validation of the PMD100 and its NOL index to detect nociception at different infusion regimen of remifentanil in patients under general anesthesia. Minerva Anestesiol. 2018;84(10):1160-1168. doi:10.23736/S0375-9393.18.12720-9

14. Meijer F, Honing M, Roor T, et al. Reduced postoperative pain using nociception level-guided fentanyl dosing during sevoflurane anaesthesia: a randomised controlled trial. Br J Anaesth. 2020;125 (6):1070-1078. doi:10.1016/j.bja.2020.07.057

15. Meijer FS, Martini CH, Broens S, et al. Nociception-guided versus standard care during remifentanil-propofol anesthesia: a randomized controlled trial. Anesthesiology. 2019;130(5):745-755. doi:10.1097/ ALN.0000000000002634

16. Shahiri TS, Richard-Lalonde M, Richebé P, Gélinas C. Exploration of the nociception level $\left(\mathrm{NOL}^{\mathrm{TM}}\right)$ index for pain assessment during endotracheal suctioning in mechanically ventilated patients in the intensive care unit: an observational and feasibility study. Pain Manag Nurs. 2020;21(5):428-434. doi:10.1016/j.pmn.2020.02.067

17. Puntillo K, Max A, Timsit JF, et al. Determinants of procedural pain intensity in the intensive care unit. The Europain ${ }^{\circledR}$ study. Am J Respir Crit Care Med. 2014;189(1):39-47. doi:10.1164/rccm.201306$11740 \mathrm{OC}$

Journal of Pain Research

\section{Publish your work in this journal}

The Journal of Pain Research is an international, peer reviewed, open access, online journal that welcomes laboratory and clinical findings in the fields of pain research and the prevention and management of pain. Original research, reviews, symposium reports, hypothesis formation and commentaries are all considered for publication. The manuscript

Submit your manuscript here: https://www.dovepress.com/journal-of-pain-research-journal
18. Gélinas C, Johnston C. Pain assessment in the critically ill ventilated adult: validation of the critical-care pain observation tool and physiologic indicators. Clin J Pain. 2007;23(6):497-505. doi:10.1097/ AJP.0b013e31806a23fb

19. Dworkin RH, Turk DC, Farrar JT, et al. Core outcome measures for chronic pain clinical trials: IMMPACT recommendations. Pain. 2005;113(1-2):9-19. doi:10.1016/j.pain.2004.09.012

20. Gélinas C. Le thermomètre d'intensité de douleur: un nouvel outil pour les patients adultes en soins critiques [The Faces Pain Thermometer: a new tool for critically ill adults]. Perspect Infirm. 2007;4(4):12-20. French.

21. Gélinas C, Joffe AM, Szumita PM, et al. A psychometric analysis update of behavioral pain assessment tools for noncommunicative, critically ill adults. AACN Adv Crit Care. 2019;30(4):365-387. doi:10.4037/aacnacc2019952

22. Sessler CN, Gosnell MS, Grap MJ, et al. The Richmond Agitation-Sedation Scale: validity and reliability in adult intensive care unit patients. Am J Respir Crit Care Med. 2002;166 (10):1338-1344. doi: $10.1164 / \mathrm{rccm} .2107138$

23. Kim HY. Statistical notes for clinical researchers: assessing normal distribution (2) using skewness and kurtosis. Restor Dent Endod. 2013;38(1):52-54. doi:10.5395/rde.2013.38.1.52

24. Kottner J, Audige L, Brorson S, et al. Guidelines for Reporting Reliability and Agreement Studies (GRRAS) were proposed. Int J Nurs Stud. 2011;48 (6):661-671. doi:10.1016/j.ijnurstu.2011.01.016

25. Bollag L, Jelacic S, Delgado Upegui C, Wu C, Richebe P. The nociception level index (NOL) response to intubation and incision in patients undergoing video-assisted thoracoscopic surgery (VATS) with and without thoracic epidural analgesia. A pilot study. F1000Res. 2018;7:875. doi:10.12688/f1000research.15 279.1

26. Martini CH, Boon M, Broens SJ, et al. Ability of the nociception level, a multiparameter composite of autonomic signals, to detect noxious stimuli during propofol-remifentanil anesthesia. Anesthesiology. 2015;123 (3):524-534. doi:10.1097/ALN.0000000000000757

27. Jildenstål P, Hallén K, Almskog K, Sand J, Warren Stomberg M. Monitoring the nociception level intraoperatively - an initial experiences. J Anaesth Intensive Care Med. 2018;7(2):555709. doi:10.19080/JAICM.2018.07.555709

28. Carr ECJ, Thomas VN, Wilson-Barnet J. Patient experiences of anxiety, depression and acute pain after surgery: a longitudinal perspective. Int J Nurs Stud. 2005;42(5):521-530. doi:10.1016/j. ijnurstu.2004.09.014

29. De Jong MM, An K, McKinley S, Garvin BJ, Hall LA, Moser DK. Using a $0-10$ scale for assessment of anxiety in patients with acute myocardial infarction. Dimens Crit Care Nurs. 2005;24(3):139-146. doi:10.1097/00003465-200505000-00008 management system is completely online and includes a very quick and fair peer-review system, which is all easy to use. Visit http:// www.dovepress.com/testimonials.php to read real quotes from published authors. 